\title{
Quantum oscillations as the tool for study of new functional materials
}

(Review Article)

\author{
V.P. Gusynin ${ }^{1}$, V.M. Loktev ${ }^{1,2}$, I.A. Luk'yanchuk ${ }^{3}$, S.G. Sharapov ${ }^{1}$, and A.A. Varlamov ${ }^{4}$ \\ ${ }^{1}$ Bogolyubov Institute for Theoretical Physics, 14-b Metrologicheskaya Str., Kiev 03680, Ukraine \\ E-mail: vloktev@bitp.kiev.ua \\ ${ }^{2}$ National Technical University of Ukraine "KPI", 37 Peremogy Ave., Kiev 03056, Ukraine \\ ${ }^{3}$ Laboratory of Condensed Matter Physics, University of Picardie Jules Verne, Amiens 80039, France \\ ${ }^{4}$ CNR-SPIN, Viale del Politecnico 1, University “Tor Vergata", I-00133 Rome, Italy
}

Received December 2, 2013

\begin{abstract}
We present an overview of our recent results on quantum magnetic oscillations in new functional materials. We begin with the Lifshitz and Kosevich approach for quasi-2D layered materials and obtain general formulas for the oscillatory parts of the grand thermodynamic potential and magnetization. Then we consider the oscillations of the Nernst-Ettingshausen coefficient which consists of thermal and magnetization parts. The difference between normal and Dirac carriers is also discussed. To conclude we consider a model for multilayer graphene which allows to calculate exactly the Berry phase which remains undetermined in the Lifshitz-Kosevich approach. The magnetic oscillations of the density of states and capacitance for different number of the carbon layers are described.
\end{abstract}

PACS: 72.15.Jf Thermoelectric and thermomagnetic effects;

72.20.Pa Thermoelectric and thermomagnetic effects;

71.70.Di Landau levels.

Keywords: quantum oscillations, magnetization, Nernst coefficient, grapheme, graphite, quasi-two-dimensional metal.

\section{Content}

1. Introduction.... .355

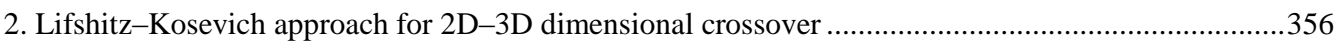

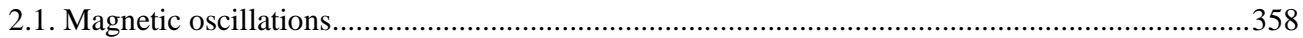

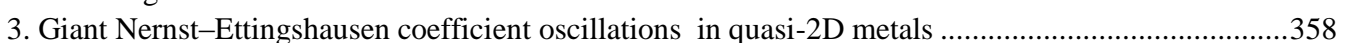

4. Exact calculation of DOS and capacitance in chiral multilayer graphene ........................................362

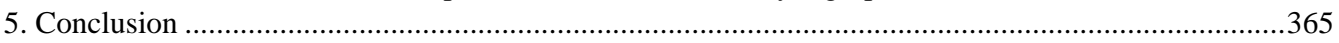

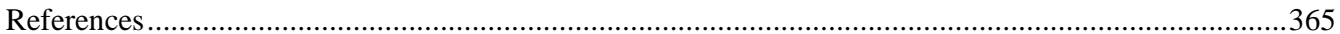

\section{Introduction}

Quantum oscillations of physical parameters of metallic and semimetallic systems in high magnetic fields is the powerful tool to study the electronic properties of new functional materials, which often are low-dimensional and with nontrivial spectra. Wealth of parameters such as electronic spectrum, scattering mechanism, geometry of Fermi surface, etc. can be extracted from the shape, period, and phase of quantum oscillations. The first experimental evidence of magnetic moment oscillations as function of ap- plied field was presented by de Haas and van Alphen in 1930 [1] and independently theoretically predicted by Landau [2] as a consequence of the electronic level quantization in magnetic field. Further theoretical description of quantum oscillations was developed in 30's in papers of Peierls [3] for 2D systems and Shoenberg [4] and Landau [5] for 3D systems.

However these studies were based on the simple metal model with quasifree electrons, described by the effective electron mass. The breakthrough in exploration of complex electron spectra occur in 1954 after the seminal work of 
Lifshitz and Kosevich (LK) [6] who generalized the Landau and Shoenberg approach for the case of arbitrary spectrum by suggestion to use the dependence of the Fermi-surface cross-section $S$ on the chemical potential $\mu: S=S(\mu)$ as the relevant parameter of the theory. In this case the cyclotron mass of the electron $m^{*}=(1 / 2 \pi) d S / d \mu$ naturally generalizes the effective electron mass for the case of nonparabolic spectra. Basing on LK theory, measurements of $S$ at different field orientations permitted to reconstruct the geometry of Fermi surface and corresponding electronic spectra for many metallic and semimetallic materials. In addition, it was recognized that complimentary information can be extracted from the study of quantum oscillations of another quantities such as longitudinal and Hall resistivity, thermopower, Nernst coefficient, etc. [7,8].

Intensive study of highly-anisotropic and low-dimensional materials, films, multilayers and superlattices posed the challenging question, how to adopt the Landau-Pierls theory for the dimensional crossover in such systems with arbitrary spectrum. In this article we present our recent results where we generalize the LK approach for systems with 2D-3D crossover in application to such exciting subject as detection and study of Dirac fermions in graphite and graphene. After introduction of the generalized LK formalism we obtain the general crossover formula for quantum oscillation of magnetization and Nernst coefficient.

Finally we consider the model of multilayer graphene which allows to calculate exactly the Berry phase which remains undetermined in the Lifshitz-Kosevich approach. The magnetic oscillations of the density of states (DOS) and capacitance for different number of the carbon layers are also studied in this model.

This work is devoted to 60th anniversary of a remarkable work of Kharkov physicists Ilya Lifshitz and Arnold Kosevich [6] which resulted in the creation of the fermiology.

\section{Lifshitz-Kosevich approach for 2D-3D dimensional crossover}

We consider the quasi-2D layered system with corrugated cylinder Fermi surface and dispersion

$$
\varepsilon(\mathbf{p})=\varepsilon_{\perp}\left(p_{\perp}\right)+2 t \sin \frac{p_{z}}{\hbar} d,
$$

where $\mathbf{p}=\left(\mathbf{p}_{\perp}, p_{z}\right)$ is the momentum $\left(p_{\perp}=\left|\mathbf{p}_{\perp}\right|\right)$ and $d$ is the distance between layers. Such a model is general enough since it accounts for the arbitrary type of dispersion $\varepsilon_{\perp}\left(p_{\perp}\right)$ in plane and allows to describe the 2D-3D dimensional crossover by variation of the interplane hopping parameter $t$ from $t_{2 D}=0$ to $t_{3 D} \sim \varepsilon_{F}$.

When the magnetic field $\mathbf{H} \| z$ is applied, the Landau quantization in perpendicular direction takes place and the quantized electronic spectrum is written as

$$
\varepsilon_{n}\left(k_{z}\right)=\varepsilon_{\perp}(n)+2 t \sin \frac{p_{z}}{\hbar} d+\sigma \mu_{B} H, \quad n=0,1, \ldots,
$$

where the last term presents the Zeeman splitting. To find $\varepsilon_{\perp}(n)$ we can use the LK approach and present the problem in terms of Fermi-surface cross-section area $S(\mu)$ of the orbital electron motion that is quantized in magnetic field in accordance to Lifshitz-Onsager condition [6,9]:

$$
S(\mu)=\left(n+\gamma_{\sigma}\right) 2 \pi \hbar \frac{e H}{c} .
$$

The correction factor to quasiclassical approximation, $\gamma_{\sigma}=\gamma+1 / 2\left(m^{*} / m\right) \sigma$, contains the orbital and spin parts [10]. The orbital part $\gamma$ was assumed in the original LK publication [6] to be in between 0 and 1 with specification that $\gamma=1 / 2$ for parabolic quasi-free electronic spectrum $\varepsilon(\mathbf{p})=p^{2} / 2 m^{*}$. More detailed calculations of Falkovsky [10] revealed that for large class of semimetallic spectra, parameter $\gamma$ can take the definite value of either 0 or $1 / 2$. Finally Mikitik and Sharlai [11] demonstrated that this factor is the topologically protected quantum number that is intimately related to so-called Berry phase and permits separate the electronic states on two classes: so-called Dirac fermions (DF) with $\gamma=0$ and normal carriers (NC) with $\gamma=1 / 2$. Proposed in [12] method of measurement of $\gamma$ via the phase of quantum oscillation permitted to detect the DF in graphite $[12,13]$ and in graphene $[14,15]$. The spin contribution $1 / 2\left(\mathrm{~m}^{*} / \mathrm{m}\right) \sigma$ with $\sigma= \pm 1$ leads to the Zeeman spin-splitting of quantum oscillation and permits to measure the electron effective mass $m^{*}$. Below we omit this factor for brevity since it can be easily reconstructed at the final stage of calculation.

As was already mentioned, the advantage of LK approach is that the quantum oscillation can be calculated in the very general form, without the detailed specification of the spectrum $\varepsilon_{\perp}\left(p_{\perp}\right)$ since the corresponding spectrum quantization can be expressed via orbital cross section (2.3) as

$$
\varepsilon_{\perp}(n)=\frac{1}{S}\left[2 \pi(n+\gamma) \frac{e \hbar}{c} H\right] .
$$

In what follows we shall use the LK formalism to calculate the quantum oscillations for spectrum (2.1) with arbitrary dependence $\varepsilon_{\perp}\left(p_{\perp}\right)$, generalizing the method that was proposed in [12] for $\varepsilon_{\perp}\left(p_{\perp}\right)=p_{\perp}^{2} / 2 m_{\perp}$.

To calculate the quantum oscillations of thermodynamical parameters we seek for the field and temperature dependences of the thermodynamic potential of the system (we assume the Boltzman constant $k_{B}=1$ )

$$
\Omega=-T \int_{-\infty}^{\infty} g(\varepsilon) \ln \left[1+\mathrm{e}^{\frac{\mu-\varepsilon}{T}}\right] d \varepsilon
$$

where $g(\varepsilon)$ is the density of states. Following [16] we present it via the imaginary part of the space-averaged electron Green function

$$
g(\varepsilon)=-\frac{1}{\pi} \operatorname{sgn}(\varepsilon-\mu) \operatorname{Im} \sum_{n, k_{z}} \frac{1}{\varepsilon-\varepsilon_{n}\left(k_{z}\right)+i \Gamma \operatorname{sgn}(\varepsilon-\mu)},
$$


where $\Gamma$ is the impurity-provided level broadening and $\varepsilon_{n}\left(k_{z}\right)$ is given by (2.4). Summation is performed over the set of one-electron quantum states that is parameterized by the Landau level quantum number $n$ and wave vector $k_{z}$. Presenting the summation over $n, k_{z}$ as

$$
\frac{1}{2 \pi} \frac{e H}{\hbar c} \int_{-\pi / d}^{\pi / d} \frac{d k_{z}}{2 \pi} \sum_{n=0}^{\infty}
$$

and using the Poisson summation over $n$

$$
\sum_{n=0}^{\infty} f(n)=\sum_{l=-\infty}^{\infty} \int_{a}^{\infty} f(\xi) \exp (2 \pi i l \xi) d \xi, \quad-1<a<0
$$

one arrives to the expression

$$
\begin{gathered}
g(\varepsilon)=\frac{1}{2 \pi} \frac{\Gamma}{\pi} \frac{e}{\hbar c} H \int_{-\pi / d}^{\pi / d} \frac{d k_{z}}{2 \pi} \times \\
\times \sum_{l=-\infty}^{\infty} \int_{-\infty}^{\infty} d \xi \frac{\exp (2 \pi i l \xi)}{\left[\varepsilon-S^{-1}\left(2 \pi(\xi+\gamma) \frac{e \hbar}{c} H\right)+2 t \cos k_{z} d\right]^{2}+\Gamma^{2}} .
\end{gathered}
$$

Here the lower limit of the last integral was extended to $-\infty$. Integration over the variable $\xi$ can be substituted by the sum of the simple pole residues, located at the points $\xi_{k}$ that are provided by condition

$$
\begin{gathered}
2 \pi\left(\xi_{k}+\gamma\right) \frac{e \hbar}{c} H=S\left(\varepsilon \pm i \Gamma+2 t \cos k_{z} d\right) \simeq \\
\simeq S(\varepsilon)+\frac{\partial S}{\partial \varepsilon} \pm i \Gamma+2 t \cos k_{z} d
\end{gathered}
$$

Finally one can obtain

$$
\begin{aligned}
& g(\varepsilon)=\frac{1}{d} \frac{1}{\pi \hbar^{2}} \frac{\partial S}{\partial \varepsilon} \int_{-\pi / d}^{\pi / d} \frac{d k_{z}}{2 \pi} \frac{1}{2} \sum_{l=-\infty}^{\infty} \exp \left(2 \pi i l\left[\frac{1}{2 \pi H} \frac{c}{e \hbar} S(\varepsilon)-\gamma\right]\right) \times \\
& \times \exp \left(-\frac{|l|}{H} \frac{c}{e \hbar}\left|\frac{\partial S}{\partial \varepsilon}\right| \Gamma\right) \exp \left(2 \pi i l\left[\frac{1}{2 \pi H} \frac{c}{e \hbar} 2 t \cos k_{z} d\right] \frac{\partial S}{\partial \varepsilon}\right) .
\end{aligned}
$$

Now, using the integral representation of Bessel function

$$
J_{0}(x)=\frac{1}{2 \pi} \int_{-\pi}^{\pi} \exp (2 \pi i x \cos \psi) d \psi
$$

integrating over $k_{z}$, and re-arranging the terms one can present $g(\varepsilon)$ in the final form

$$
\begin{gathered}
g(\varepsilon)=\frac{1}{d} \frac{m^{*}}{\pi \hbar^{2}}+\frac{2}{\pi} \frac{1}{d} \frac{m^{*}}{\hbar^{2}} \times \\
\times \sum_{l=1}^{\infty} \exp \left(-\pi l \frac{\beta \Gamma}{\mu_{B} H}\right) J_{0}\left(\frac{\beta t}{\mu_{B} H} l\right) \cos \left(2 \pi l\left[\frac{c}{e \hbar} \frac{S(\varepsilon)}{2 \pi H}-\gamma\right]\right),
\end{gathered}
$$

where Bohr magneton $\mu_{B}=e \hbar / 2 m c$, and $\beta$ is defined in Table 1.

Table 1. Parameters of general LK formalism for the particular cases of normal carriers (NC) and Dirac fermions (DF) with parabolic and linear energy dispersions $p_{\perp}^{2} / 2 m_{\perp}$ and $v\left|p_{\perp}\right|$ correspondingly

\begin{tabular}{c|c|c}
\hline \hline Parameter & $\mathrm{NC}$ & $\mathrm{DF}$ \\
\hline$\varepsilon_{\perp}\left(p_{\perp}\right)$ & $p_{\perp}^{2} / 2 m_{\perp}$ & $v\left|p_{\perp}\right|$ \\
$\varepsilon_{\perp}(n)$ & $\hbar \omega_{c}\left(n+\frac{1}{2}\right)$ & $\pm\left(2 \mu \hbar \omega_{c} n\right)^{1 / 2}$ \\
$m^{*}=\frac{1}{2} \frac{d S}{d \mu}, \beta=\left|m^{*}\right| / m$ & $2 \pi m_{\perp} \mu$ & $\pi \mu^{2} / v^{2}$ \\
$\gamma_{\sigma}$ & $\frac{1}{2}+\frac{1}{2} \frac{m_{\perp}}{m} \sigma$ & $\frac{1}{2} \frac{\mu}{m v^{2}} \sigma$ \\
$\omega_{c}=\frac{e H}{m^{*} c}, \hbar \omega_{c}=2 \beta^{-1} \mu_{B} H$ & $e H / c m_{\perp}$ & $e v^{2} H / c \mu$ \\
$H_{c}=\frac{c}{e \hbar} \frac{S(\mu)}{2 \pi}$ & $\frac{1}{2} \beta \frac{\mu}{\mu_{B}}$ & $\frac{1}{4} \frac{\mu}{m v^{2}} \frac{\mu}{\mu_{B}}$ \\
$\lambda=\pi^{2} \frac{\beta T}{\mu_{B} H}$ & $4 \pi m_{\perp} t d^{2}$ & $4 \pi t d^{2} \mu / v^{2}$ \\
\hline \hline$p_{z}^{2}=2 \frac{d S}{d \mu} t d^{2}$ & - & - \\
\hline
\end{tabular}

The thermodynamical potential $\Omega$ contains the nonoscillating, $\Omega_{c}$, and oscillating, $\Omega$, parts. The first contribution is easily calculated from (2.4) and (2.9) by integration by parts

$$
\Omega_{c}=-\frac{1}{2 \pi^{2}} \frac{1}{d} \frac{1}{\hbar^{2}} \int_{0}^{\mu} S(\varepsilon) d \varepsilon+O(T) .
$$

For the oscillating part $\tilde{\Omega}$ we expand $S(\varepsilon)$ in vicinity of $\mu$ as $S(\varepsilon) \simeq S(\mu)+2 \pi m^{*}(\varepsilon-\mu)$ and, after integration by parts, one obtains 


$$
\begin{gathered}
\tilde{\Omega}=-\frac{1}{d} \frac{1}{\pi} \frac{e}{\hbar c} H T \sum_{l=1}^{\infty} \frac{1}{l} \exp \left(-\pi l \frac{\beta \Gamma}{\mu_{B} H}\right) J_{0}\left(\frac{\beta t}{\mu_{B} H} l\right) \times \\
\times \operatorname{Im}\left[\exp i\left(2 \pi l\left[\frac{c}{e \hbar} \frac{S(\mu)}{2 \pi H}-\gamma\right]\right) \frac{1}{\pi} \int_{0}^{\infty} \frac{\exp 2 \pi i l \frac{c}{e \hbar} \frac{m^{*}}{H}(\varepsilon-\mu)}{1+\exp \left(\frac{\varepsilon-\mu}{T}\right)}\right] d \varepsilon .
\end{gathered}
$$

The last integral can be calculated by expansion of the lower limit to $-\infty$ (valid for $T \ll \mu)$ ) and by using the relation

$$
\int_{-\infty}^{\infty} \frac{\exp i \alpha y}{1+\exp y} d y=-\frac{i \pi}{\sinh \alpha \pi}
$$

Finally one finds

$$
\tilde{\Omega}=\frac{m^{*}}{2 \pi \hbar^{2}} \frac{\hbar^{2} \omega_{c}^{2}}{\pi^{2}} \frac{1}{2} \sum_{l=1, \sigma= \pm 1}^{\infty} \frac{\psi(\lambda l)}{l^{2}} \Phi_{l \sigma}^{\prime} \mu, H
$$

with $\psi(\lambda l)=\lambda l / \sinh \lambda l$, and

$$
\begin{gathered}
\Phi_{l \sigma} \mu, H=\Phi_{l \sigma}^{\prime}+i \Phi_{l \sigma}^{\prime \prime}= \\
=J_{0}\left(2 \pi l \frac{2 t}{\hbar \omega_{c}}\right) \exp \left\{\left[-\frac{\Gamma}{\hbar \omega_{c}}+i\left(\frac{c}{e \hbar} \frac{S(\mu)}{2 \pi H}-\gamma_{\sigma}\right)\right] 2 \pi l\right\} .
\end{gathered}
$$

Here we reconstructed the spin variable $\sigma= \pm 1$. The dimensional crossover is driven by parameter $x=2 t /\left(\hbar \omega_{c}\right): x \ll 1$ in quasi-2D case and $x \gg 1$ in $3 \mathrm{D}$ case.

\subsection{Magnetic oscillations}

Knowledge of the field and temperature dependences of oscillating part of thermodynamical potential $\tilde{\Omega}(H, T)$ permits to calculate the quantum oscillations of various thermodynamical parameters of the system, and, in particularly, oscillating magnetization

$$
\begin{gathered}
\tilde{M}=-\left(\frac{\partial \tilde{\Omega}}{\partial H}\right)_{\mu, T}= \\
=-\frac{1}{(2 \pi)^{2}} \frac{e}{\hbar c} \frac{S(\mu)}{\pi^{2} m^{*}} \frac{1}{2} \sum_{l=1, \sigma= \pm 1}^{\infty} \frac{\psi(\lambda l)}{l^{2}} \Phi_{l \sigma}^{\prime \prime}(\mu, H) .
\end{gathered}
$$

Using the limit expansions $J_{0}(x)^{x \ll 1} \simeq 1, \quad J_{0}(x)^{x \gg 1} \simeq$ $\simeq(2 / \pi x)^{1 / 2} \cos (x-\pi / 4)$ we, following [18], present the quantum oscillation of magnetization in $2 \mathrm{D}(x \ll 1)$ and 3D $(x \gg 1)$ cases as

$$
\begin{gathered}
\tilde{M}_{2 D}=-\frac{1}{4 \pi^{3}} \frac{\mu_{B}}{a_{B}^{2}}\left(\frac{S / m^{*}}{\mathrm{Ry}}\right) \times \\
\times \sum_{l=1, \sigma= \pm 1}^{\infty} \frac{\psi(\lambda l)}{l} \mathrm{e}^{-\beta \frac{\pi \Gamma}{\mu_{B} H} \sin \left(\beta \frac{S / m^{*}}{2 \mu_{B} H}-2 \pi \gamma_{\sigma}\right) l}
\end{gathered}
$$

and

$$
\begin{gathered}
\tilde{M}_{3 D}=-\frac{\sqrt{2 \pi}}{8 \pi^{3}} \frac{\mu_{B}}{a_{B}^{3}}\left(\frac{S / m^{*}}{\mathrm{Ry}}\right)\left(\frac{\mu_{B} H}{\mathrm{Ry}}\right)^{1 / 2}\left|\frac{\partial^{2} S}{\partial p_{z}^{2}}\right|^{-1 / 2} \times \\
\times \sum_{l=1, \sigma= \pm 1}^{\infty} \frac{\psi(\lambda l)}{l^{3 / 2}} \mathrm{e}^{-\pi \beta \frac{\Gamma}{\mu_{B} H} l} \sin \left(\beta \frac{S / m^{*}}{2 \mu_{B} H} l-2 \pi \gamma_{\sigma} l \pm \frac{\pi}{4}\right),
\end{gathered}
$$

where 2D magnetization is normalized per unit of the layer area and 3D magnetization per unit of volume. The atomic units: Bohr radius $a_{B}=\hbar^{2} /\left(m e^{2}\right)$ and Rydberg energy $\mathrm{Ry}=m e^{4} / 2 \hbar^{2}$ were used to simplify the dimensional analysis of the resulting expressions.

It is interesting to note, that Eq. (2.15) can be written in a simple form at low temperatures $\pi^{2} \beta T<\mu_{B} H$ when $\psi(\lambda l) \approx 1$ :

$$
\begin{gathered}
\tilde{M}_{2 D}=-\frac{1}{2 \pi^{3}} \frac{\mu_{B}}{a_{B}^{2}}\left(\frac{S / m^{*}}{\mathrm{Ry}}\right) \times \\
\times \frac{1}{2} \sum_{\sigma= \pm 1} \arctan \frac{\sin \left(\beta \frac{S / m^{*}}{2 \mu_{B} H}-2 \pi \gamma_{\sigma}\right)}{\exp \left(\beta \frac{\pi \Gamma}{\mu_{B} H}\right)-\cos \left(\beta \frac{S / m^{*}}{2 \mu_{B} H}-2 \pi \gamma_{\sigma}\right)} .
\end{gathered}
$$

In case of NC this expression was obtained in [16] and in case of DF in [17]. For more detailed analysis of dimension- and temperature-induced crossover of magnetic oscillations we refer to publication [18].

\section{Giant Nernst-Ettingshausen coefficient oscillations in quasi-2D metals}

In this section written basing mainly on the results of Ref. 19, we will apply the general relations obtained above in order to explain the giant Nernst-Ettingshausen (NE) coefficient oscillations observed recently on the graphene samples [20,21]. Let us start from recalling of the basic notions.

The Nernst-Ettingshausen effect in metals [22] can be considered as a thermoelectric counterpart of the Hall effect. It consists of the induction of an electric field $E_{y}$ normal to the mutually perpendicular magnetic field $H_{z}$ and temperature gradient $\nabla_{x} T$. All electric circuits are supposed to be broken: $J_{x}=J_{y}=0$ and heat flow along 
$y$ axis to be absent (adiabatic conditions). Quantitatively the NE effect is characterized by the NE coefficient

$$
v=\frac{E_{y}}{\left(-\nabla_{x} T\right) H_{z}},
$$

which varies by several orders of magnitude in different materials ranging from about $7 \mathrm{mV} /(\mathrm{K} \cdot \mathrm{T})$ in bismuth (where the effect has been observed by NE for the first time in 1886) up to $10^{-5} \mathrm{mV} /(\mathrm{K} \cdot \mathrm{T})$ in some metals [23].

NE effect remained poorly understood until 1948 when Sondheimer [24], basing on the classical Mott formula for the thermoconductivity tensor, calculated $v$ for a degenerated electron system, linking it to the energy derivative of the Hall angle $\theta=\sigma_{x y} / \sigma_{x x}$ which allowed to reveal a correlation between NE and Hall effects. Within this model $v$ was found to be independent on the magnetic field in weak fields and to decrease as $\mathrm{H}^{-2}$ in the region of classically strong fields, when the cyclotron frequency $\omega_{c}$ is larger then the inverse electron mean free time $\tau^{-1}$.

In 1959 Bergeron and co-authors experimentally observed the giant oscillations of the NE coefficient $v$ in strong magnetic fields in zinc [25]: the phenomenon apparently related to crossing of the electronic Fermi energy by Landau levels (LL). Simular to de Haas-van Alphen (dHvA) oscillations of magnetization and Shubnikov-de Haas $(\mathrm{SdH})$ oscillations of conductivity, in the NE oscillations the corresponding quantizing fields $H=H_{n}$ are given by Lifshitz-Onsager condition (2.3). Later, in 1964, Obraztsov [26] suggested that so-called magnetization currents (i.e., electric currents induced due to inhomogeneous distribution of magnetization in the sample) can contribute supplementary to the NE effect.

Very recently, the NE effect has been measured [20,21] and theoretically analyzed $[27,28]$ in graphene. Surprisingly, it has been found that $v$ changes its sign at $H=H_{n}$ in graphene while it has maxima at these intersections in zinc [25] and bismuth [29]. Zhu et al. [30] demonstrated that such untypical behavior of $v(H)$ observed in graphene is not reproduced in graphite. They concluded that piling of multiple graphene layers leads to a topological phase transition in the spectrum of charge carriers, so that graphite behaves as a 3D crystal despite of its apparent structural anisotropy and of similarity of its electronic properties to those of graphene.

Another challenging property of quantum oscillations is the possibility to distinguish between two types of charge carriers, having already mentioned topologically different parameter $\gamma$ [11]: $\gamma=1 / 2$ for the NC with the parabolic 2D dispersion and linear LL quantization and $\gamma=0$ for the DF having the linear two-branch spectrum and $\sim n^{1 / 2}$ LL quantization.

Below we use a simple thermodynamic approach to the description of the NE effect which allows linking the oscillations of the NE coefficient to the presented above oscilla- tions of the chemical potential. Both thermal (Sondheimer) and magnetization contributions to the Nernst coefficient are evaluated analytically for a quasi-2D electronic system with either parabolic or Dirac spectrum. In the 2D limit for the Dirac spectrum we recover the behavior of the NE coefficient observed in graphene $[20,21]$ while the recent data of Zhu et al. [30] on graphite are fitted better assuming a quasi-2D character of the system. Very interestingly, the amplitude of the NE oscillation is found to decrease as a function of the Fermi energy in system of Dirac fermions, while it increases with Fermi energy for carriers having a parabolic $2 \mathrm{D}$ dispersion.

As it was mentioned above the NE coefficient is measured in the absence of the electric current flowing through the system along the temperature gradient. The system is at thermal equilibrium and can be characterized by a constant electrochemical potential $\mu+e \varphi$, where $\mu$ is supposed to be the temperature dependent chemical potential and $\varphi$ is the electrostatic potential. In the thermodynamic approximation [31,32], the effect of the temperature gradient is reduced to appearance of an effective electrostatic potential and, henceforth, an electric field in $x$ direction $E_{x}=\nabla \mu / e$. In this way, the problem is reduced to the classical Hall problem, which allows to obtain the thermal contribution to the NE coefficient:

$$
v^{\text {therm }}=\frac{\sigma_{x x}}{e^{2} n c} \frac{d \mu}{d T},
$$

where $\sigma_{x x}$ is the diagonal component of the conductivity tensor, $n$ is the concentration of carriers. This simple formula reproduces the result of Sondheimer for a normal metal [32].

An additional contribution to NE coefficient appearing due to the spatial dependence of magnetization in the sample [26] can be found from the Ampere law: $\mathbf{j}^{\text {mag }}=(c / 4 \pi) \nabla \times \mathbf{B}$, where $\mathbf{j}^{\text {mag }}$ is the magnetization current density, the magnetic induction $\mathbf{B}=\mathbf{H}+4 \pi \mathbf{M}, \mathbf{H}$ is the spatially homogeneous external magnetic field, $\mathbf{M}$ is the magnetization, which can be temperature and, henceforth, coordinate dependent. One can readily express the magnetization current as $j_{y}^{\mathrm{mag}}=-c(d M / d T) \nabla T$ and the corresponding contribution to the electric field in $y$ direction (Nernst field) as $E_{y}^{\mathrm{mag}}=\rho_{y y} j_{y}^{\mathrm{mag}}$, where $\rho_{y y}$ is the diagonal component of the resistivity tensor. The "magnetization" contribution to the NE coefficient reads

$$
v^{\mathrm{mag}}=\frac{c \rho_{y y}}{H} \frac{d M}{d T} .
$$

The Eqs. (3.2) and (3.3) reveal the essential physics of Nernst oscillations in the quantizing magnetic fields. In particular, one can see that the NE coefficient is dependent on the diagonal components of conductivity and resistivity tensors. Their oscillations as a function of magnetic field constitute the $\mathrm{SdH}$ effect. In graphene and graphite the 
giant Nernst oscillations have been observed in the regime where the SdH effect is negligibly weak [20,21,30]. This is why one should attribute the giant NE coefficient oscillations to the remaining factors in the Eqs. (3.2) and (3.3), namely to the temperature derivatives of the chemical potential and magnetization, $d \mu / d T$ and $d M / d T$, respectively. Remarkably, to evaluate these quantities no supplementary knowledge of the transport properties of the system is needed. These derivatives can be expressed in terms of the thermodynamic potential of the system $\Omega(\mu, H, T)$ as

$$
\frac{d \mu}{d T}=\frac{\partial^{2} \Omega}{\partial T \partial \mu}\left(\frac{\partial^{2} \Omega}{\partial \mu^{2}}\right)_{T}^{-1} \text { and } \frac{d M}{d T}=\frac{\partial^{2} \Omega}{\partial T \partial H} .
$$

The corresponding expression for the oscillating (denoted by tilde) part of $\Omega$ is given by Eq. (2.12). One can present it in the LK form [6], using the Fermi-surface cross section $S(\mu)$ at $p_{z}=0$, the electron cyclotron mass $m^{*}$, the cyclotron frequency $\omega_{c}$, and the phase $\gamma$ as the parameters that do not depend on the particular form of $\varepsilon_{\perp}\left(p_{\perp}\right)$. Their specific values for the parabolic and Dirac spectra are given in the Table 1 above. Below we omit the index $\sigma$ implying the spin averaging.

In the experimental configuration of the NE effect, the number of particles $n$ is fixed, so that [16]

$$
n=-\left(\frac{\partial \Omega(\mu)}{\partial \mu}\right)_{H, T}=2 \frac{S(\mu)}{2 \pi \hbar^{2}}-\left(\frac{\partial \tilde{\Omega}(\mu)}{\partial \mu}\right)_{H, T}=\text { const. }
$$

This relation implicitly determines the dependence of $\mu$ on $H$ and $T$ for the given $n$. In the present derivation we assume a Lorentzian broadening of LL with a constant $\Gamma$. Such approximation can be justified for $\omega_{c} \ll \varepsilon_{F}$ in the case of 3D system. In 2D systems it is expected to be valid only in the low-field regime $\omega_{c} \lesssim \tau^{-1}$. The oscillating parts of the chemical potential and magnetization can be expressed using Eq. (3.4) as

$$
\frac{d \tilde{\mu}}{d T}=-\frac{\operatorname{Im} \Xi^{\{1\}}}{1+2 \operatorname{Re} \Xi^{\{0\}}}, \quad \frac{d \tilde{M}}{d T}=\frac{n}{H} \frac{d \tilde{\mu}}{d T},
$$

where

$$
\Xi^{\{\alpha\}}=\frac{1}{2} \sum_{l=1, \sigma= \pm 1}^{\infty} \psi^{(\alpha)}(\lambda l) \Phi_{l \sigma}\left(\varepsilon_{F}, H\right)
$$

and $\psi^{(\alpha)}(x)$ is the derivative of the order of $\alpha=0,1$ of the function $\psi$. One can see from Eqs. (3.3) and (3.6) that the NE coefficient oscillates proportionally to the derivative of magnetization over temperature. This shows an important link between NE and dHvA oscillations, which is universal and independent on the dimensionality of the system and of the type of carriers.

It is convenient to express the NE coefficient as

$$
v=v^{\text {therm }}+v^{\text {mag }}=v_{0}(H)+\tilde{v}(H)
$$

with $v_{0}(H)$ and $\tilde{v}(H)$ being the background and the oscillating parts. The background part can be evaluated in the Drude approximation as [32]

$$
v_{0}(H)=\frac{\pi^{2} \tau}{6 m^{*} c}\left(\frac{T}{\varepsilon_{F}}\right) \frac{1}{1+\left(\omega_{c} \tau\right)^{2}} .
$$

Taking into account the magnetization currents leads to the correction of the order of $\left(\varepsilon_{F} \tau\right)^{-2}$ with respect to Sondheimer result described by Eq. (3.9).

The oscillating part of the Nernst coefficient can be written using Eqs. (3.2), (3.3) and (3.6) as

$$
\tilde{v}(H)=-2 \pi \kappa(H) \frac{\operatorname{Im} \Xi^{\{1\}}}{1+2 \operatorname{Re} \Xi^{\{0\}}},
$$

with

$$
\kappa(H)=\frac{\sigma_{x x}(H)}{e^{2} n c}+\frac{c n \rho_{x x}(H)}{H^{2}} .
$$

In the Drude approximation for NC

$$
\kappa_{\text {Drude }}(H)=\frac{\tau}{m^{*} c} \frac{1}{\left(\omega_{c} \tau\right)^{2}} \frac{1+2\left(\omega_{c} \tau\right)^{2}}{1+\left(\omega_{c} \tau\right)^{2}}
$$

Equation (3.10) describes oscillations of the NE effect in the most general form. It is valid for any type of the dispersion $\varepsilon_{\perp}\left(p_{\perp}\right)$ if $T, t \ll \mu$.

The $2 D$ case: graphene. We start analysis of the Eq. (3.10) from the pure 2D case when $t=0$. In the lowtemperature limit $2 \pi^{2} T<\hbar \omega_{c}$ in Eq. (2.12) $\lambda \ll 1$, hence $\psi(\lambda l) \approx 1-\left(\lambda^{2} l^{2}\right) / 6$. For $m^{*}<0.02 m$ and $H=10 \mathrm{~T}$ (typical in graphene experiments) this yields $T<10 \mathrm{~K}$. Since $m^{*} \ll m$ we neglect also the Zeeman splitting, assuming that $\gamma_{\sigma}=\gamma=0$ for NC and $\gamma_{\sigma}=\gamma=1 / 2$ for DF. The series $\Xi^{\{0\}}$ and $\Xi^{\{1\}}$ in Eq. (3.10) in this case can be summed exactly which gives

$$
\tilde{v}^{(2 D)}(\mu, H)=\frac{2 \pi^{3} T}{3 \hbar \omega_{c}} \frac{\kappa(H) \sin 2 \pi\left[\frac{c}{e \hbar} \frac{S(\mu)}{2 \pi H}-\gamma\right]}{\cosh \frac{2 \pi \Gamma}{\hbar \omega_{c}}-\cos 2 \pi\left[\frac{c}{e \hbar} \frac{S(\mu)}{2 \pi H}-\gamma\right]} .
$$

In the experimental configuration corresponding to the measurement of the NE effect in graphene, the number of particles $n$ is fixed. 
Hense the chemical potential in the $2 \mathrm{D}$ case can be determined from the equation

$$
n=2 \frac{S(\mu)}{(2 \pi \hbar)^{2}}+\frac{m^{*}}{\hbar^{2}} \frac{\hbar \omega_{c}}{\pi^{2}} \arctan \frac{\sin 2 \pi\left(\frac{c}{e \hbar} \frac{S(\mu)}{2 \pi H}-\gamma\right)}{\mathrm{e}^{\frac{2 \pi \Gamma}{\hbar \omega_{c}}}-\cos 2 \pi\left(\frac{c}{e \hbar} \frac{S(\mu)}{2 \pi H}-\gamma\right)} .
$$

This equation can be inverted for $S(\mu)$ :

$$
\frac{c}{e \hbar} \frac{S(\mu)}{2 H}=\pi^{2} \frac{\hbar c}{e} \frac{n}{H}-\arctan \frac{\sin 2 \pi\left(\pi \frac{\hbar c}{e} \frac{n}{H}-\gamma\right)}{\mathrm{e}^{\frac{2 \pi \Gamma}{\hbar \omega_{c}}}+\cos 2 \pi\left(\pi \frac{\hbar c}{e} \frac{n}{H}-\gamma\right)} .
$$

Equation (3.15) yields the dependence $\mu(n, H)$. Substituting it to Eq. (3.13) after some cumbersome algebra one can find the oscillating part of the Nernst coefficient explicitly:

$$
\tilde{v}^{(2 D)}(n, H)=\frac{2 \pi^{3}}{3} \frac{T}{\hbar \omega_{c}} \frac{\kappa(H)}{\sinh \frac{2 \pi \Gamma}{\hbar \omega_{c}}} \sin 2 \pi\left(\pi \frac{\hbar c}{e} \frac{n}{H}-\gamma\right),
$$

that is a strongly oscillating function. It crosses zero at the intersections of LL and chemical potential, given by the condition $H=H_{k \sigma}$ defined by (2.3). The field depended factor $\kappa(H)$ is governed by magnetoresistance and is given by Eq. (3.11). At $\omega_{c} \tau \leq 1$ where $\mathrm{SdH}$ oscillations are small, $\kappa(H)$ can be roughly estimated using the Drude approximation (3.12). In particular, approaching the limit $\omega_{c} \tau \sim 1$ and assuming $\Gamma \sim \hbar / 2 \tau$ we obtain that $\kappa(H) \sim \tau /\left(m^{*} c\right)$ and the amplitude of NE oscillations is giant in comparison with the background: $\tilde{v}^{(2 D)} \sim\left(\varepsilon_{F} / \hbar \omega_{c}\right) v_{0}$. At higher fields $\omega_{c} \tau>1$, in the quantum Hall regime, the shape of oscillations of the NE coefficient is affected by strong variation of the magnetoresistance and Dingle temperature. This can be taken into account by substitution of the corresponding magnetoresistance and Dingle temperature field dependence into Eqs. (3.10), (3.11).

The profiles of 2D NE oscillation as function of $H$ and $n$ for DF and NC given by Eq. (3.16) are presented in Fig. 1. Both our theory for DF and experiment in graphene [20,21] show a sine-like profile of the signal whose amplitude slightly decreases with increasing $n$. This tendency contradicts to the earlier theoretical predictions of the classical Mott formula [20] that has been de-
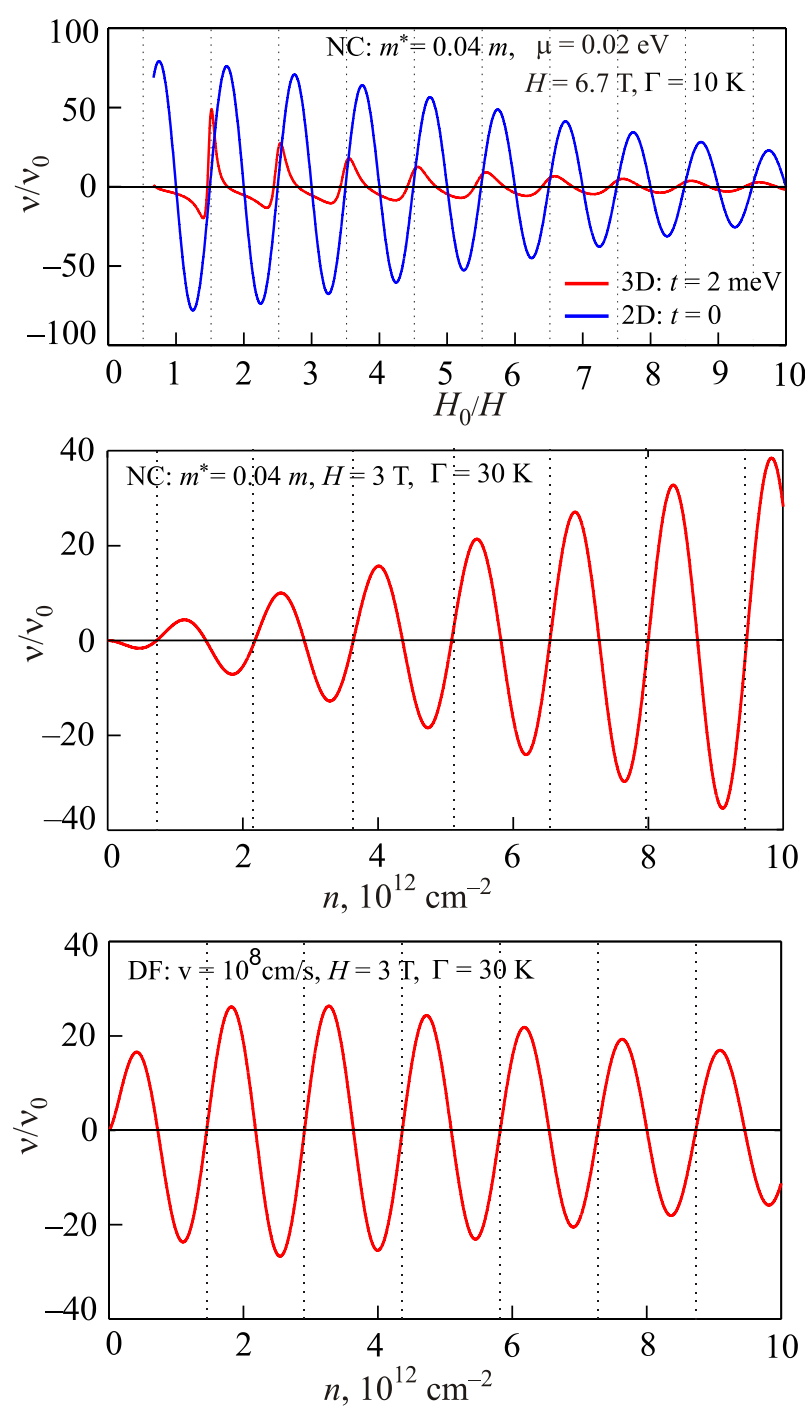

Fig. 1. (Color online) The normalized NE oscillations $v / v_{0}$ as function of the inverse magnetic field $H_{0} / H$ and carriers concentration $n$ for $\mathrm{NC}$ and DF. The dependence $v\left(H^{-1}\right)$ for DF has the same profile as for $\mathrm{NC}$ but shifted by half period. The vertical lines show the quantization condition (2.3).

rived for a Boltzmann gas of electrons. In contrast, the amplitude of NE oscillations increases with increasing $n$ for the NC in a qualitative agreement with the Mott formula.

Quasi-2D and $3 D$ cases. In order to describe the NE effect in the general quasi-2D case when $t \neq 0$ the Bessel function in the Eq. (2.13) should be taken into account. The sums (3.7) can be reduced to the integrals by means of the Poisson transformation. Then integration can be done analytically resulting in

$$
\Xi^{\{0\}}=\frac{1}{2} \sum_{\substack{k=-\infty \\ \sigma= \pm 1}}^{\infty} \frac{1}{2 \pi\left[\delta_{k \sigma}^{2}(H)+\frac{4 t^{2}}{\hbar^{2} \omega_{c}^{2}}\right]^{1 / 2}}-\frac{1}{2}
$$


and

$$
\Xi^{\{1\}}=-\frac{1}{6} \frac{T}{\hbar \omega_{c}} \frac{1}{2} \sum_{\substack{k=-\infty \\ \sigma= \pm 1}}^{\infty} \frac{\delta_{k \sigma}(H)}{\left[\delta_{k \sigma}^{2}(H)+\frac{4 t^{2}}{\hbar^{2} \omega_{c}^{2}}\right]^{3 / 2}},
$$

where

$$
\delta_{k \sigma}(H)=\frac{\Gamma}{\hbar \omega_{c}}-i \frac{c}{\hbar e} \frac{S}{2 \pi}\left(H^{-1}-H_{k \sigma}^{-1}\right) .
$$

The NE coefficient is obtained by substitution of the Eqs. (3.17) and (3.18) to Eq. (3.10). Resonances at $i \delta_{k \sigma}(H)= \pm 2 t /\left(\hbar \omega_{c}\right)$ in $\tilde{v}(H)$ appear when the chemical potential crosses the quantized slices of maximal (minimal) cross sections of the corrugated cylinder Fermi surface $S_{\max (\min )}=S \pm 4 \pi t m^{*}$.

In the wide quasi-2D interval $t<\left(\hbar \omega_{c}\right)^{2} / \Gamma$ the behavior of $\tilde{v}^{(q 2 D)}(H)$ close to $H=H_{k \sigma}$ can be studied selecting in (3.17) and (3.18) only the resonant terms. With growth of $t$ the positions of zeros shift from $\operatorname{Im} \delta_{k \sigma}(H)=0$ to $\operatorname{Im} \delta_{k \sigma}(H)= \pm 2 t /\left(\hbar \omega_{c}\right)$. The superposition of two (for $S_{\max }$ and $S_{\min }$ ) series of resonances leads to the beats in $\tilde{v}(H)$ oscillations.

In the 3D limit $t>\left(\hbar \omega_{c}\right)^{2} / \Gamma$, Re $\Xi^{\{0\}} \ll 1$, so that $\Xi^{\{0\}}$ can be neglected in the denominator of Eq. (3.10). In the vicinity of $H=H_{k \sigma}$ one finds

$$
\tilde{v}^{(3 D)}(H)=\mp \frac{\pi}{12} \frac{T \kappa(H)}{\left(t \hbar \omega_{c}\right)^{1 / 2}} \operatorname{Re} \frac{1}{\left[\frac{2 t}{\hbar \omega_{c}} \pm i \delta_{k}(H)\right]^{3 / 2}} .
$$

We assumed here the constant $\mu$ and neglected Zeeman splitting, taking $\delta_{k, \pm 1}=\delta_{k}$. The resonances in $\tilde{v}(H)$ described by Eq. (3.19) have the form of asymmetric spikes with $\left|\tilde{v}^{(3 D)}\right|_{\max } /\left|\tilde{v}^{(3 D)}\right|_{\min } \simeq 3.4$ as shown in Fig. 1. In the Drude approximation the amplitude

$$
\left|\tilde{v}^{(3 D)}\right|_{\max } \simeq 0.29 \frac{\varepsilon_{F}}{\Gamma} \frac{\hbar \omega_{c}}{(t \Gamma)^{1 / 2}} v_{0}(H)
$$

is giant if $\left(\varepsilon_{F} / \Gamma\right) \hbar \omega_{c} /(t \Gamma)^{1 / 2}>1$.

For 2D systems our calculations are valid for magnetic fields $\omega_{c} \lesssim \tau^{-1}$ where one can neglect the quantum Hall oscillations of conductivity. At higher fields the approach of Girvin and Jonson [33], based on the generalized Mott formula for the thermopower tensor for 2D systems, seems to be more relevant. In 3D case the range of applicability of our theory is given by $\omega_{c} \ll \varepsilon_{F}$. Recently Bergman and Oganesyan [28] extended the approach of Ref. 33 to calculate the off-diagonal thermoelectric conductivity $\alpha_{x y}$ for a 3D system at $\omega_{c} \sim \varepsilon_{F}$. Although $\alpha_{x y}$ constitute only the part of NE coefficient $v=-\left(\rho_{x x} \alpha_{x y}+\rho_{x y} \alpha_{y y}\right) / H$, they reproduce quite well the sawtooth dependence of $v(H)$, measured in graphite [30] with the characteristic $\left(H_{k}-H\right)^{-1 / 2}$ divergences at resonances.

\section{Exact calculation of DOS and capacitance in chiral multilayer graphene}

In order to treat the single-layer, bilayer graphene [34] and chiral multilayer graphene (neglecting the trigonal warping effects) with $N \geq 2$ layers [35-38] simultaneously we start from the Hamiltonian given in a unified form as

$$
\begin{gathered}
H_{N}=\sum_{\xi, \sigma} \int d^{2} \mathbf{x} \Psi_{\xi \sigma}^{+}(\mathbf{x}) \times \\
\times\left[-\xi^{N} a_{N}\left(\begin{array}{cc}
0 & \left(\hat{p}_{-}\right)^{N} \\
\left(\hat{p}_{+}\right)^{N} & 0
\end{array}\right)+\xi\left(\begin{array}{cc}
\Delta & 0 \\
0 & -\Delta
\end{array}\right)\right] \Psi_{\xi \sigma}(\mathbf{x}),
\end{gathered}
$$

where $\hat{p}_{ \pm}=\hat{p}_{x} \pm i \hat{p}_{y}, \hat{\mathbf{p}}$ is the canonical momentum operator, $a_{N}=\gamma_{1}\left(v_{F} / \gamma_{1}\right)^{N}, \quad v_{F}=\sqrt{3} / 2 t a \sim 10^{6} \mathrm{~m} / \mathrm{s}$ is the Fermi velocity in graphene, $a \approx 2.46 \AA$ is the lattice constant, $\gamma_{0} \approx 3.16 \mathrm{eV}$, and $\gamma_{1} \approx 0.39 \mathrm{eV}$ are tight-binding parameters responsible for the in-layer nearest-neighbor and interlayer hopping, respectively. While for $N=1$ the Hamiltonian is valid for momenta $p \ll 1 / a$, for $N \geq 2$ the low-energy effective Hamiltonian (4.1) can be utilized for momenta $p$ up to $p_{W}=\gamma_{1} / v_{F}$. The two-component spinor field $\Psi_{\xi \sigma}$ carries the valley $\left(\xi= \pm\right.$ for the $K$ and $K^{\prime}$ valleys, respectively) and spin $(\sigma= \pm)$ indices. For the ABC-stacked multilayer graphene, the low-energy electron states are located only on the outermost layers which we will denote as layers 1 and $N$ in what follows. Further, we use the standard convention for wave functions: $\Psi_{+\sigma}^{T}=\left(\psi_{+A_{1}}, \psi_{+B_{N}}\right)_{\sigma}$, whereas $\Psi_{-\sigma}^{T}=\left(\psi_{-B_{N}}, \psi_{-A_{1}}\right)_{\sigma}$. Here $A_{1}$ and $B_{N}$ correspond to those sublattices in the outermost layers 1 and $N$, respectively, which are relevant for the low-energy dynamics. Obviously, for $N=1$ the sublattices belong to the same layer. In the Hamiltonian (4.1) we also included the mass (gap) term which describes interlayer asymmetry between on-site energy $\xi \Delta$ of the atoms, $A_{1}$ and $B_{1}$, on the first layer and $-\xi \Delta$ of the atoms, $A_{N}$ and $B_{N}$, on the outermost layer.

In the presence of magnetic field $H$ perpendicular to the layers the energies of LL are [39] (see also Refs. 40 and 41)

$$
\epsilon_{n \xi}=-\xi \Delta, \quad n=0,1, \ldots, N-1
$$

and

$\epsilon_{n}= \pm M_{n}, M_{n}=\sqrt{\Delta^{2}+\hbar^{2} \omega_{c N}^{2} n(n-1) \cdots(n-N+1)}, n \geq N$,

where $\hbar \omega_{c N}$ is the cyclotron energy of multilayer expressed via the Landau scale $L=\sqrt{2 \hbar v_{F}^{2}|e H| / c} \approx$ $\approx 36.3 \sqrt{H[\mathrm{~T}]} \mathrm{meV}$ for monolayer graphene and the hop- 
ping $\gamma_{1}$ as follows: $\hbar \omega_{c N}=a_{N}(\sqrt{2} \hbar / l)^{N}=L^{N} / \gamma_{1}^{N-1}$, where $l=\sqrt{\hbar c /|e H|}$ is the magnetic length.

The DOS is written as

$$
\begin{gathered}
D(\epsilon)=\frac{1}{\pi l^{2}}\{N \delta(\epsilon-\Delta)+N \delta(\epsilon+\Delta)+ \\
\left.+2 \sum_{n=N}^{\infty}\left[\delta\left(\epsilon-M_{n}\right)+\delta\left(\epsilon+M_{n}\right)\right]\right\} .
\end{gathered}
$$

Here we neglected the Zeeman splitting, because it is smaller than the cyclotron energy (for example, $\left.\hbar \omega_{c 2}=3.4 \mathrm{meV} B[\mathrm{~T}]\right)$. To calculate the DOS we follow Ref. 17 and write

$$
\begin{gathered}
D(\epsilon)=\frac{2|\epsilon|}{\pi l^{2}}\left[N \delta\left(\epsilon^{2}-\Delta^{2}\right)+2 \sum_{n=N}^{\infty} \delta\left(\epsilon^{2}-M_{n}^{2}\right)\right]= \\
=\frac{1}{\pi l^{2}} \operatorname{sgn}(\epsilon) \frac{d}{d \epsilon}\left\{\theta\left(\epsilon^{2}-\Delta^{2}\right)+\right. \\
\left.+2 \sum_{n=1}^{\infty} \theta\left[\epsilon^{2}-\Delta^{2}-\hbar^{2} \omega_{c N}^{2} n(n+1) \ldots(n+N-1)\right]\right\} .
\end{gathered}
$$

Using the Poisson summation formula

$$
\frac{1}{2} F(0)+\sum_{n=1}^{\infty} F(n)=\int_{0}^{\infty} d x F(x)+2 \operatorname{Re} \sum_{k=1}^{\infty} \int_{0}^{\infty} d x F(x) \mathrm{e}^{2 \pi i k x},
$$

we find the sum over the LL

$$
\begin{gathered}
D(\epsilon)=\frac{2}{\pi l^{2}} \operatorname{sgn}(\epsilon) \frac{d}{d \epsilon} \times \\
\times\left\{\theta\left(\epsilon^{2}-\Delta^{2}\right)\left[\frac{N-1}{2}+x_{0}(\epsilon, N)+\sum_{k=1}^{\infty} \frac{\sin \left(2 \pi k x_{0}(\epsilon, N)\right)}{\pi k}\right]\right\},
\end{gathered}
$$

where $x_{0}(\epsilon, N)$ is the largest and the only positive root of the algebraic equation

$$
\prod_{k=0}^{N-1}(x+k)=\frac{\epsilon^{2}-\Delta^{2}}{\hbar^{2} \omega_{c N}^{2}} .
$$

Making the shift $x=z-(N-1) / 2$ this equation can be rewritten as

$$
z \prod_{k=1}^{(N-1) / 2}\left(z^{2}-k^{2}\right)=\frac{\epsilon^{2}-\Delta^{2}}{\hbar^{2} \omega_{c N}^{2}}
$$

for odd $N$, and

$$
\prod_{k=1}^{N / 2}\left[z^{2}-\left(\frac{2 k-1}{2}\right)^{2}\right]=\frac{\epsilon^{2}-\Delta^{2}}{\hbar^{2} \omega_{c N}^{2}}
$$

for even $N$. Hence for DOS we obtain

$$
D(\epsilon)=\frac{2}{\pi l^{2}} \operatorname{sgn}(\epsilon) \times
$$

$$
\times \frac{d}{d \epsilon}\left\{\theta\left(\epsilon^{2}-\Delta^{2}\right)\left[z_{0}(\epsilon, N)+\sum_{k=1}^{\infty} \frac{(-1)^{k(N-1)}}{\pi k} \sin \left(2 \pi k z_{0}(\epsilon, N)\right)\right]\right\},
$$

where $z_{0}(\epsilon, N)$ is the largest root of the above equations. Note the presence of the factor $(-1)^{N-1}$ in oscillations which is a reflection of the presence of the Berry phase. Indeed, we can write

$$
\frac{1-N}{2}=\frac{1}{2}-\frac{\Phi_{\text {Berry }}}{2 \pi},
$$

where the Berry phase $\Phi_{\text {Berry }}=\pi N$ for graphene with $N$ layers. Thus our model calculation allows to find the value of $\gamma=\bmod [(N-1) / 2]$, where $\bmod [x]$ is the shorthand notation for the fractional part $x$ modulo 1 . In particular, one can see that for $N=1$ we obtain $\gamma=0[10,11]$ which allows to distinguish Dirac carriers in monolayer graphene. Moreover, even the opening of the gap $\Delta$ in the quasiparticle spectrum does not change this value of $\gamma$.

In weak magnetic fields (quasiclassical regime) Eqs. (4.8), (4.9) both give the largest root

$$
z_{0}(\epsilon, N)=\frac{c}{2 \hbar|e H|}\left(\frac{\epsilon^{2}-\Delta^{2}}{a_{N}^{2}}\right)^{1 / N} .
$$

On the other hand, from the dispersion relation in the absence of a magnetic field, $\epsilon=\sqrt{\Delta^{2}+a_{N}^{2}\left(p^{2}\right)^{N}}$, we find that the Fermi-surface cross section is

$$
S(\varepsilon)=\pi p^{2}=\pi\left(\frac{\varepsilon^{2}-\Delta^{2}}{a_{N}^{2}}\right)^{1 / N}
$$

and the value $z_{0}(\epsilon, N)$ coincides with the argument $c S(\epsilon) /(2 \pi \hbar|e H|)$ in oscillating functions in Eqs. (2.9) and (2.12). For example, the DOS (4.11) for weak magnetic fields can be written in the form

$$
D(\epsilon)=\frac{m^{*}(\epsilon)}{\pi \hbar^{2}}\left[1+2 \sum_{k=1}^{\infty}(-1)^{k(N-1)} \cos \left(2 \pi k \frac{c S(\epsilon)}{2 \pi \hbar|e H|}\right)\right]
$$

where $m^{*}(\epsilon)=(1 / 2 \pi)[\partial S(\epsilon) / \partial|\epsilon|]$. It coincides with the corresponding DOS $g(\epsilon)$ given by Eq. (2.9) if we set the coupling $t=0, \Gamma=0$ and omit the factor $1 / d$.

The knowledge of zero temperature DOS is completely sufficient to write down the finite $T$ thermodynamic potential and other thermodynamic quantities. The DOS and its oscillations can be experimentally found by measuring the 
quantum capacitance $C[42,43]$ which is proportional to the thermally smeared DOS and is given by

$$
C(\mu)=e^{2} \int_{-\infty}^{\infty} d \epsilon D(\epsilon)\left[-n_{F}^{\prime}(\epsilon)\right]
$$

where $n_{F}(\epsilon)=1 /[\exp (\epsilon-\mu) / T+1]$ is the Fermi distribution. Assuming that the presence of impurities broadens the LL to the Lorentzian form with a field-independent width $\Gamma$ we can write

$$
\begin{aligned}
& D(\epsilon)=\frac{\Gamma}{\pi^{2} l^{2}}\left\{\frac{N}{(\epsilon-\Delta)^{2}+\Gamma^{2}}+\frac{N}{(\epsilon-\Delta)^{2}+\Gamma^{2}}+\right. \\
& \left.+2 \sum_{n=1}^{\infty}\left[\frac{1}{\left(\epsilon-M_{n}\right)^{2}+\Gamma^{2}}+\frac{1}{\left(\epsilon+M_{n}\right)^{2}+\Gamma^{2}}\right]\right\} .
\end{aligned}
$$

Using the representation

$$
\frac{\Gamma}{\epsilon^{2}+\Gamma^{2}}=\int_{0}^{\infty} d t \mathrm{e}^{-\Gamma t} \cos (\epsilon t)
$$

and the integral

$$
\begin{aligned}
& \int_{-\infty}^{\infty} d \epsilon\left[-n_{F}^{\prime}(\epsilon)\right] \cos \left[\left(\epsilon-M_{n}\right) t\right]= \\
& =\cos \left[\left(M_{n}-\mu\right) t\right] \frac{\pi t T}{\sinh (\pi t T)},
\end{aligned}
$$

we present the capacitance in the form convenient for numerical calculations:

$$
\begin{gathered}
C(\mu)=\frac{e^{2}}{\pi^{2} l^{2}}\{N I(\mu-\Delta)+N I(\mu+\Delta)+ \\
\left.\quad+2 \sum_{n=1}^{\infty}\left[I\left(\mu-M_{n}\right)+I\left(\mu+M_{n}\right)\right]\right\},
\end{gathered}
$$

where the function

$$
\begin{aligned}
I(x) & =\int_{0}^{\infty} d t \mathrm{e}^{-\Gamma t} \cos (x t) \frac{\pi T t}{\sinh (\pi T t)}= \\
& =\frac{1}{2 \pi T} \operatorname{Re} \Psi^{\prime}\left(\frac{1}{2}+\frac{\Gamma-i x}{2 \pi T}\right)
\end{aligned}
$$

can be expressed in terms of digamma function $\Psi(x)$. In Fig. 2 we show the dependence $C(\mu)$ for $N=1,2,3$ and for a particular case of zero gap $\Delta=0$. Comparing the behavior of the DOS as the number of layers $N$ increases
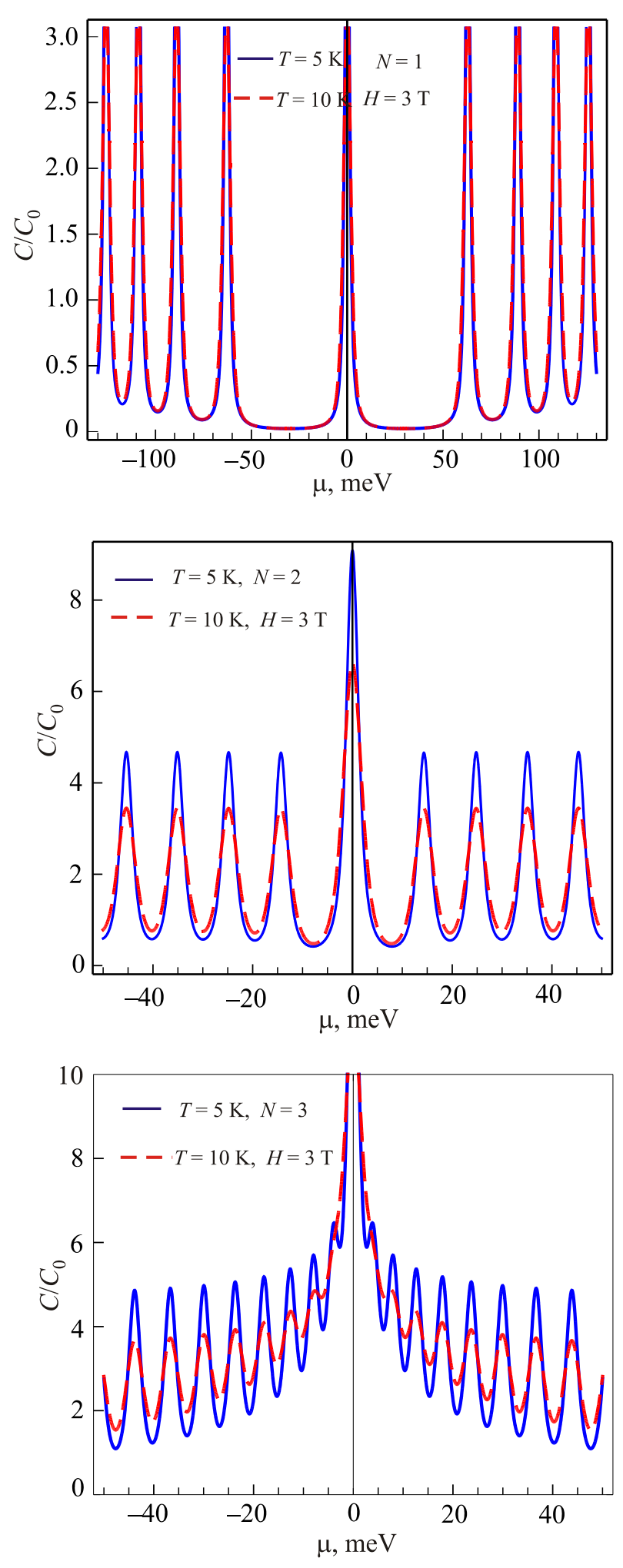

Fig. 2. (Color online) Capacitance (per unit area in units of $C_{0}=2.4 \mu \mathrm{F} / \mathrm{cm}^{2}$ ) of multilayer graphene as a function of the chemical potential $\mu$ for $H=3 \mathrm{~T}, \Gamma=1 \mathrm{meV}, \Delta=0, T=5 \mathrm{~K}$ (solid (blue) curve) and $T=10 \mathrm{~K}$ (dashed (red) curve). Top panel: monolayer graphene, $N=1$; middle panel: bilayer graphene, $N=2$; and bottom panel: trilayer graphene, $N=3$. 
one can see that the contribution of the lower LL enhances because the levels are getting denser near zero energy. Furthermore, the DOS envelop function itself reflects the behavior of the zero field DOS given by

$$
D(\epsilon)=\frac{|\epsilon|\left(\epsilon^{2}-\Delta^{2}\right)^{(1-N) / N}}{\pi N \hbar^{2} a_{N}^{2}} \theta(|\epsilon|-\Delta) .
$$

The strength of the peak corresponding to the zero energy LL also increases as the number of layers grows which reflects the degeneracy of this level (see Eq. (4.4)).

\section{Conclusion}

We applied the LK formalizm to consider the dimensional 3D-2D crossover of quantum oscillations in layered systems with an arbitrary electronic spectrum and derived the general formula for oscillating thermodynamic potential in this case. Such an approach permits to consider the quantum oscillations of various thermodynamic parameters in a unify way. In particularly, we presented the explicit crossover formula for $\mathrm{dHvA}$ oscillation of magnetization and specified it for the 2D and 3D cases. Then, we considered quantum oscillations of the NE coefficient and obtained an analytical expression for 2D case with an arbitrary electron dispersion. This expression was used to explain the recent experimental results in graphene. In addition it can be used to distinguish between NC and DF in $2 \mathrm{D}$ sysetms. We showed that the giant oscillations of the NE coefficient, predicted and observed in a 2D case (graphene), decrease significantly as the spectrum acquires a 3D character (graphite). We described analytically also the shape of NE oscillations and predicted that in all cases the NE oscillations are proportional to the temperature derivative of the dHvA oscillations.

In the model of chiral multilayer graphene we studied the magnetic oscillations of the density of states and capacitance for different number of the carbon layers and determined the Berry phase which remains undetermined in the LK approach.

\section{Acknowledgments}

This work is supported partially by the European FP7 program, Grant No. SIMTECH 246937. The work of V.P.G., V.M.L. and S.G.Sh. is supported also by the grant STCU \#5716-2 "Development of Graphene Technologies and Investigation of Graphene-based Nanostructures for Nanoelectronics and Optoelectronics". V.P.G. and S.G.Sh. acknowledge the support of the joint Ukrainian-Russian SFFR-RFBR grant No. F53.2/028. The work of IL was done in frame of International Training Network FP7-ITNNOTEDEV.
1. W.J. de Haas and P.M. van Alphen, Proc. Netherlands Roy. Acad. Sci. 33, 680 (1930).

2. L.D. Landau, Z. Phys. 64, 629 (1930).

3. R. Peierls, Z. Phys. 80, 763 (1933); Z. Phys. 81, 186 (1933).

4. D. Shoenberg, Proc. Roy. Soc. A 170, 341 (1939).

5. L.D. Landau, Proc. Roy. Soc. A 170, 341 (1939) (Appendix to article of D. Shoenberg [4]).

6. I.M. Lifshitz and A.M. Kosevich, Dokl. Akad. Nauk SSSR 96, 963 (1954); Zh. Eksp. Teor. Fiz. 29, 730 (1955).

7. I.M. Lifshitz, M.Ya. Azbel', and M.I. Kaganov, Electron Theory of Metals, Consultants Bureau, New York (1973).

8. D. Shoenberg, Magnetic Oscillations in Metals, Cambridge University Press (1984).

9. L. Onsager, Philos. Mag. 43, 1006 (1952).

10. L.A. Falkovsky, Zh. Eksp. Teor. Fiz. 49, 609 (1965) [Sov. Phys. JETP 22, 609 (1966)].

11. G.P. Mikitik and Yu.V. Sharlai, Phys. Rev. Lett. 82, 2147 (1999).

12. I.A. Luk'yanchuk and Y. Kopelevich, Phys. Rev. Lett. 93, 166402 (2004).

13. I.A. Luk'yanchuk and Y. Kopelevich, Phys. Rev. Lett. 97, 256801 (2006).

14. K.S. Novoselov, A.K. Geim, S.V. Morozov, D. Jiang, M.I. Katsnelson, I.V. Grigorieva, S.V. Dubonos, and A.A. Firsov, Nature 438, 197 (2005).

15. Y. Zhang, Y.-W. Tan, H.L. Stormer, and Philip Kim, Nature 438, 201 (2005).

16. T. Champel and V.P. Mineev, Philos. Mag. B 81, 55 (2001).

17. S.G. Sharapov, V.P. Gusynin, and H. Beck, Phys. Rev. B 69 , 075104 (2004).

18. I.A. Luk'yanchuk, Fiz. Nizk. Temp. 37, 56 (2011) [Low Temp. Phys. 37, 45 (2011)].

19. I.A. Luk'yanchuk, A.A. Varlamov, and A.V. Kavokin, Phys. Rev. Lett. 107, 016601 (2011).

20. Y.M. Zuev, W. Chang, and P. Kim, Phys. Rev. Lett. 102, 096807 (2009).

21. J.G. Checkelsky and N.P. Ong, Phys. Rev. B 80, 081413(R) (2009).

22. A. Von Ettingshausen and W. Nernst, Wied. Ann. 29, 343 (1886).

23. K. Behnia, M.A. Méasson, and Y. Kopelevich, Phys. Rev. Lett. 98, 076603 (2007).

24. E.H. Sondheimer, Proc. R. Soc. London 193, 484 (1948).

25. C.J. Bergeron, C.G. Grenier, and J.M. Reynolds, Phys. Rev. Lett. 2, 40 (1959).

26. Yu.N. Obraztsov, Sov. Phys. Solid State 6, 331 (1964).

27. V.P. Gusynin and S.G. Sharapov, Phys. Rev. B 73, 245411 (2006).

28. D.L. Bergman and V. Oganesyan, Phys. Rev. Lett. 104, 066601 (2010).

29. K. Behnia, M.A. Méasson, and Y. Kopelevich, Phys. Rev. Lett. 98, 166602 (2007).

30. Z. Zhu, H. Yang, B. Fauque, Y. Kopelevich, and K. Behnia, Nature Physics 6, 26 (2010).

31. M.N. Serbyn, M.A. Skvortsov, A.A. Varlamov, and V. Galitski, Phys. Rev. Lett. 102, 067001 (2009). 
32. A.A. Varlamov and A.V. Kavokin, Europhys. Lett. 86, 47007 (2009).

33. M. Jonson and S. Girvin, Phys. Rev. B 29, 1939 (1984).

34. J. Cserti, Phys. Rev. B 75, 033405 (2007).

35. H. Min and A.H. MacDonald, Phys. Rev. B 77, 155416 (2008).

36. Y. Barlas, K. Yang, and A.H. MacDonald, Nanotechnology 23, 052001 (2012).

37. R. Côté, M. Rondeanu, A.-M. Gagnon, and Yafis Barlas, Phys. Rev. B 86, 125422 (2012).

38. M. Nakamura and L. Hirasawa, Phys. Rev. B 77, 045429 (2008).
39. M. Koshino and E. McCann, Phys. Rev. B 80, 165409 (2009).

40. S. Yuan, Rafael Roldán, and M.I. Katsnelson, Phys. Rev. B 84, 125455 (2011).

41. R. Côté and M. Barrette, Preprint arXiv:1310.7551.

42. L.A. Ponomarenko, R. Yang, R.V. Gorbachev, P. Blake, A.S. Mayorov, K.S. Novoselov, M.I. Katsnelson, and A.K. Geim, Phys. Rev. Lett. 105, 136801 (2010).

43. G.L. Yu, R. Jalil, B. Belle, A.S. Mayorov, P. Blake, F. Schedin, S.V. Morozov, L.A. Ponomarenko, F. Chiappini, S. Wiedmann, U. Zeitler, M.I. Katsnelson, A.K. Geim, K.S. Novoselov, and D.C. Elias, Proc. Natl. Acad. Sci. USA 110, 3281 (2013). 